\title{
Public health interventions to improve maternal nutrition during pregnancy: a nationally representative study of iron and folic acid consumption and food supplements in India
}

\author{
Prashant Kumar Singh ${ }^{1}$, Ritam Dubey ${ }^{1}$, Lucky Singh ${ }^{2, *} \odot{ }^{*}$, Chandan Kumar ${ }^{3}$, \\ Rajesh Kumar Rai ${ }^{4}$ and Shalini Singh ${ }^{5}$ \\ 'Division of Preventive Oncology, ICMR National Institute of Cancer Prevention and Research, Noida, India: \\ ${ }^{2}$ ICMR National Institute of Medical Statistics, New Delhi 110029 , India: ${ }^{3}$ Department of Policy Studies, TERI School of \\ Advanced Studies, New Delhi, India: ${ }^{4}$ Society for Health and Demographic Surveillance, Suri, West Bengal, India: \\ 5 ICMR National Institute of Cancer Prevention and Research, Noida, India
}

Submitted 14 August 2019: Final revision received 19 February 2020: Accepted 13 March 2020: First published online 1 July 2020

\begin{abstract}
Objective: Despite a reduction in maternal mortality in recent years, a high rate of anaemia and other nutrient inadequacies during pregnancy pose a serious threat to mothers and their children in the Global South. Using the framework of the WHOCommission on Social Determinants of Health, this study examines the socioeconomic, programmatic and contextual factors associated with the consumption of iron and folic acid (IFA) tablets/syrup for at least $100 \mathrm{~d}$ (IFA100) and receiving supplementary food (SF) by pregnant women in India.

Design: We analysed a nationally representative cross-sectional survey of over 190898 ever-married women aged 15-49 years who were interviewed as part of the National Family Health Survey (NFHS) conducted during 2015-16, who had at least one live birth preceding 5 years of the survey.

Setting: All twenty-nine states and seven union territories of India.

Participants: Ever-married women aged 15-49 years.

Results: Less than one-third of women were found to be consuming IFA100, and a little over half received SF during their last pregnancy. The consumption of IFA100 was likely to improve with women's education, household wealth, early and more prenatal visits, and in a community with high pregnancy registration. Higher parity, early and more prenatal visits, contact with community health workers during pregnancy, belonging to a poor household and living in an aggregated poor community and rural area positively determine whether a woman might receive SF during pregnancy.

Conclusions: Continuous monitoring and evaluation of provisioning IFA and SF in targeted groups and communities is a key to expanding the coverage and reducing the burden of undernutrition during pregnancy.
\end{abstract}

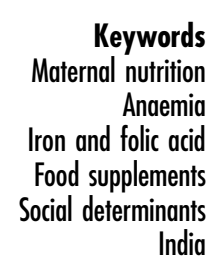

Maternal and child undernutrition remains one of the significant causes of millions of deaths and disability every year ${ }^{(1)}$. Low- and middle-income countries (LMIC) in particular face the brunt of this global crisis where pregnant women have a much higher risk of iron deficiency anaemia $(\text { IDA })^{(2)}$, inadequacy of vital micronutrients, proteins and energy $^{(3)}$. This results in a high proportion of adverse pregnancy outcomes such as low birth weight and stunting in infants born in these regions ${ }^{(4)}$.
During pregnancy, the requirements of energy, protein and essential micronutrients (vitamins and minerals) increase not only to maintain the mother's health but also to support optimal physical and brain development of the foetus $^{(3)}$. Public health interventions in many countries target diet, nutritional supplements, multiple micronutrients during pregnancy and have shown beneficial effects on reducing preterm births and low birth weights ${ }^{(5)}$. In the Indian context, evidence suggests that the top two risk 
factors causing disability and premature deaths are related to malnutrition and poor $\operatorname{diet}^{(6)}$. Moreover, despite the considerable reductions in maternal and child mortality in the last decade, nutritional indicators associated with women and children remain deplorable ${ }^{(7)}$.

To address undernutrition among pregnant women, the federal Indian government focused on two national programmes, namely, provisioning of iron and folic acid (IFA) tablets/syrup and supplementary food (SF) to women during pregnancy ${ }^{(8)}$ with the objective of reducing the burden of $\mathrm{IDA}^{(9)}$, and improving the overall nutrition. Under the National Iron+ Initiative, pregnant and lactating women are prescribed a dose of $100 \mathrm{mg}$ elemental $\mathrm{Fe}$ and $500 \mu \mathrm{g}$ folic acid, with a prescribed regime of one tablet daily for $100 \mathrm{~d}$ (IFA100), starting after the first trimester, at 14-16 weeks of gestation, and repeated up to $100 \mathrm{~d}$ postpartum $^{(9)}$. On the other hand, the Mid-day Meal Scheme (MMS), the Integrated Child Development Services (ICDS), the Mahatma Gandhi National Rural Employment Guarantee Scheme (MGNREGS) and the Public Distribution System (PDS) were devised to reduce undernutrition by providing supplementary food (SF) directly or by offering economic support to secure food for the household ${ }^{(8)}$. A further step was taken with the introduction of the National Food Security Act (NFSA) 2013, and priority was given to food and nutrition security among women ${ }^{(8)}$. The NFSA outlines that women are to receive supplementary nutrition - which consists of one meal that meets basic nutritional standards, free of charge, during pregnancy and 6 months after childbirth - through the local anganwadi centres. Eligible women are entitled to take home ration, which supplements $2510 \mathrm{~kJ}$ (600 kcal) of energy with 18$20 \mathrm{~g}$ of $\operatorname{protein}^{(8)}$.

Several studies have analysed IFA and micronutrient uptake among pregnant women in Africa ${ }^{(10)}$, Indonesia ${ }^{(11)}$, Cambodia ${ }^{(12)}$ and India ${ }^{(13)}$. A few studies from India suggest that the effectiveness of IFA and SF intervention to reduce undernutrition remains far from expected, although both programmes seem to have helped in improving coverage ${ }^{(9,14)}$.

An often-repeated query is about the access and adequate utilisation of healthcare programmes. In the present context, whether and to what extent the pregnant women, as beneficiaries of public nutritional interventions, are receiving the benefits of these nutritional health programmes remains unclear. Moreover, considering the huge regional differences in culture, healthcare infrastructure and socioeconomic status, it would be useful to explore the links and associations at multiple levels leading to the utilisation of maternal nutrition programmes in India. Existing empirical literature does not provide adequate evidence in this direction, as most of the studies have attempted to capture individual-level factors responsible for public health nutrition interventions, ignoring the role of programme and contextual determinants in such a diverse country ${ }^{(9,15)}$. Building upon the present gaps in scientific literature, this study examines the individual, programmatic and contextual factors associated with the utilisation of two important interventions related to maternal nutrition, including IFA and SF, in India. Moreover, the results of this study could guide the evaluation of such programmes implemented in other LMIC.

\section{Methods}

\section{Conceptual framework}

This study follows the WHO-Commission on Social Determinants of Health (CSDH) framework ${ }^{(16)}$ to understand the individual, socioeconomic, programmatic and contextual factors responsible for the uptake of IFA100 and SF. The framework illustrates how social, economic and political mechanisms promote socioeconomic positions, whereby populations are stratified according to income, education, gender, race/ethnicity and other factors. These socioeconomic positions, in turn, shape specific determinants of health status or health services, and reflect people's place within social hierarchies. Following the $\mathrm{CSDH}$ framework, the variables constructed to understand the individual, programmatic and contextual factors can be categorised as below.

1. Socioeconomic factors - These include maternal age at birth of the referenced children, women's education, parity, gender composition of living children, caste/ social group, wealth quintile and religion.

2. Programmatic factors - These include the timing of prenatal care in terms of months of pregnancy, total number of prenatal visits, meeting community healthcare providers during pregnancy and the place where antenatal care (ANC) was received.

3. Compositional/contextual factors - These include the proportion of illiterate and poor population in the community, that is, the primary sampling unit (PSU), and the proportion of registered pregnancies in the district along with place and region of residence.

A detailed description of these factors is given in the following sub-section related to explanatory variables.

\section{Data and sample}

This study is based on the fourth round of the National Family Health Survey (NFHS) conducted during 2015-16 across all twenty-nine states and seven union territories of India ${ }^{(7)}$. The NFHS is an adaptation of the Demographic and Health Survey of India. A two-stage stratified random sampling was used to draw samples from both urban and rural areas. Villages in rural areas and Census Enumeration Blocks (CEB) in urban areas served as the PSU or clusters. A total of 28586 PSU were selected across India. The household response rate was $98 \%$, whereas it was $97 \%$ among women. A detailed description 
of survey design, sample and stratifications is available online at http://rchiips.org/nfhs/NFHS-4Report.shtml. The study sample includes 190898 ever-married women aged 15-49 years who had at least one live birth in the 5 years preceding the survey. The sample represents a generalisable characteristic of Indian women as the distribution of the sample across social group/caste, religion and place of residence (rural/urban) followed the Indian Census 2011 statistics (see online supplementary material, Supplemental Table S1).

\section{Outcome variables}

The consumption of a recommended dose of IFA (i.e. at least one tablet or equivalent amount of syrup per day for $100 \mathrm{~d}$ ) and receiving SF during pregnancy are considered the outcome variables of this study. In NFHS-4, women were asked to report the number of days they took IFA tablets during pregnancy. Similarly, women respondents were asked to provide information on whether they received SF during pregnancy if they responded to receiving any benefit from the anganwadi/ICDS centres. The WHO strongly recommends daily oral IFA supplements during pregnancy as part of prenatal care to reduce the risk of low birth weight, maternal anaemia and Fe deficiency $^{(17)}$. Pregnant and lactating mothers are entitled to receive cooked food served at anganwadi centres on a daily basis or take-home food provisions as per the guidelines of the Ministry of Women and Child Development, Government of India. The SF provided to pregnant and lactating women at the centre is supposed to include $2510 \mathrm{~kJ}(600 \mathrm{kcal})$ of energy and $18-20 \mathrm{~g}$ of protein per day in the form of micronutrient-fortified food and/or energydense food as take-home ration ${ }^{(18)}$.

\section{Explanatory variables}

\section{Individual/household factors}

Women's characteristics analysed were age, education (categorised based on the highest years of schooling attained) and parity. The gender composition of living children to women was considered a proxy variable to assess the behavioural aspect of women, reflecting their earnestness towards pregnancy care. Women's affiliation to the social group - documented as caste (categorised as Scheduled Castes (SC), Scheduled Tribes (ST), Other Backward Classes (OBC) and Others (who are not eligible to receive incentives from the government)) and religion (categorised as Hindu, Muslim and Others) - is also considered along with their household economic status (measured in terms of wealth quintiles: poorest, poorer, middle, richer and richest). Previous studies have found that these variables had considerable influence in determining the interventions addressing maternal nutrition during pregnancy ${ }^{(19,20)}$.

\section{Programme-dependent factors}

During early pregnancy, the health system contacts at various platforms are evidenced to influence the utilisation of other health services in continuum. Thus, the utilisation of these services is determined by important programmedependent factors - contact of women with healthcare providers (at home or at other places) during pregnancy, time of first prenatal care or registration with the health facility (in terms of month of pregnancy: within or in the 3rd month, 4th-5th month, and in the 6th month or later), number of prenatal care visits and place where prenatal care was received. Early pregnancy registration with the health facility or community health workers was significantly associated with the consumption of IFA supplements, and a health worker is more likely to visit those pregnant women who were registered for prenatal care $^{(13)}$.

These health system contacts also work as a proxy for nutrition education and counselling (NEC) given during pregnancy. Studies around community-based distribution (CBD) of IFA supplements and NEC have shown these to be effective strategies in increasing the consumption of IFA tablets among pregnant women ${ }^{(21)}$. The CBD is a strategy to provide IFA supplements to women directly through community channels, such as private pharmacies, community health centres, village health workers, community health workers, community volunteers or community gatherings for health education sessions ${ }^{(22)}$.

\section{Compositional/contextual factors}

The associations between shared spatial attributes in the neighbourhood and health outcomes/behaviours are well documented in public health literature ${ }^{(23,24)}$. The theories of social capital ${ }^{(25,26)}$, including social networks and relationships $^{(25,26)}$, community awareness and diffusion ${ }^{(27)}$, provide a framework for understanding the mechanism through which communities may influence the utilisation of healthcare services. The proportion (\%) of illiterate persons in a PSU and the proportion (\%) of the poor in a PSU, as well as the type of residential place (rural or urban), were included in the study to reflect the impact of shared community/neighbourhood on the use of health nutrition services. In NFHS, one PSU is more often the size of a standard village (with $>40-50$ households) in rural areas, and Indian villages are still a cohesive unit in terms of socialisation. A close urban neighbourhood would also incur more or less a similar aggregate impact on the utilisation of health services by individuals in that community.

In India, the district serves as the lowest programme implementation unit in the overall administrative hierarchy. Literature suggests that the role of individual, household and community factors differs from one geographic setting to another ${ }^{(28)}$. A few area-specific contextual factors, such as the proportion (\%) of pregnancy cases registered in 
districts, and state groups, are also included in the study. The Government of India has designated eight states (Bihar, Chhattisgarh, Jharkhand, Madhya Pradesh, Orissa, Rajasthan, Uttarakhand, Uttar Pradesh) into the 'Empowered Action Group' (EAG) and Assam under a group of 'High Focus States' (HFS), which account for about $48 \%$ of India's population.

\section{Analytical strategies}

Owing to the hierarchical structure of the data and the nature of factors, especially compositional or contextual factors, namely individuals (level 1) grouped in clusters (level 2) and then in districts (level 3), a multilevel framework was adopted to analyse the influence of covariates at individual/household, community and district levels on the utilisation of nutritional interventions during pregnancy. From a statistical perspective, this structure of data (grouped data) implies a violation of the assumption of independence among observations within the same second- and third-level units, that is, individuals living in the same community and district ${ }^{(29)}$. To address the structure of data and the binary nature of outcome variables, we applied a multilevel binary logistic regression model with random intercept specification that includes random intercepts and fixed slopes at specified levels ${ }^{(30)}$. A major impetus for examining the role of contexts in explaining health variations comes from the advances in quantitative methods, in particular those related to multilevel statistical methods ${ }^{(31)}$.

Data analysis was performed with Stata (version 14$)^{(32)}$ using the 'xtmelogit' command for a logistic linear random intercept model. Bivariate associations between plausible factors and outcome variables were checked using $\chi^{2}$ tests. Factors found to be significant in the $\chi^{2}$ test were included in the multivariate analyses. Before applying multivariate analyses, the intercept-only model (referred to as the 'empty model') was estimated for both outcomes. Intraclass correlation coefficients (ICC) were estimated to assess heterogeneity at the community and district levels. ICC refers to the proportion of total variance that is accounted for by the group level. The likelihood ratio test was incorporated to see whether the estimated variances were significant. A separate analysis between HFS and nonHigh Focus States (NHFS) was conducted to examine the differences in the determinants of IFA consumption and receiving SF during pregnancy.

In addition, based on the full model estimated for both outcomes, district-specific (posterior) residuals were estimated and mapped using ArcGIS suite. Residual mapping is an extremely useful application of multilevel models to present the model-based implications for policy and programme implementation, or the observed heterogeneity at the level of administrative decision-making and implementation ${ }^{(33)}$.

\section{Results}

\section{Differentials in the uptake of iron and folic acid and supplementary food}

Table 1 presents the proportion (\%) of women consuming IFA and receiving SF by select characteristics. All the listed characteristics had a statistically significant association with both outcome measures. Less than one-third of women (30\%) reported consuming IFA, while slightly more than half (52\%) confirmed receiving SF during pregnancy. The consumption of IFA100 was relatively higher among women who were educated, lived in an urban area and belonged to a socioeconomically advantageous background. Further, the consumption of IFA was higher among women who did early pregnancy registration (38\%) and those who received ANC services at least once by any doctor $(40 \%)$ or at higher-level facilities. On the other hand, women who were in contact with community health workers or received prenatal services at least once from community health workers or at ICDS centres or subcentres received $\mathrm{SF}$ in higher proportions.

District-wise pattern in the utilisation of IFA (Fig. 1(a)) and SF (Fig. 1(c)) shows considerable variations. Out of 640 districts, 156 and 44 had $<15 \%$ coverage of IFA and $\mathrm{SF}$, respectively. Moreover, only eighty-seven districts had $>55 \%$ coverage of IFA, while the number of corresponding districts receiving SF was 333. Districts with a lower coverage of both services were largely concentrated in the north, east and western states of India.

\section{Factors associated with the use of iron and folic acid tablets/syrup for at least $100 \mathrm{~d}$ and supplementary food}

The results of the random intercept-only model (Table 2) infer that there were considerable variations in the utilisation of IFA (26\% at district level and $44 \%$ at PSU level) and receipt of SF (31\% at district level and 53\% at PSU level). Findings of a multivariate logistic regression analysis suggest that the use of IFA during pregnancy was significantly determined by the women's age at childbirth (positively associated), education (positively associated), parity (negatively associated), economic status (positively associated), caste and religious affiliation (Table 3 ).

As far as programme-level factors are concerned, women who registered their pregnancy in the first trimester (OR 1.33, $95 \%$ CI 1.09, 1.62), who were contacted by healthcare providers both at home and outside (OR 1.29, $95 \%$ CI 1.23, 1.35), who had four or more ANC visits (OR 1.74, 95\% CI 1.68, 1.79) or who had at least one ANC visit at a higher-level facility showed higher odds of IFA consumption compared to women who received no ANC, who did not meet any healthcare provider during pregnancy or who received ANC at home. Women in the HFS had nearly $30 \%$ less probability of using IFA100 
Table 1 Proportion (\%) of women consuming iron and folic acid for at least $100 \mathrm{~d}$ (IFA100) and receiving supplementary food (SF) during pregnancy in the 5 years preceding the survey by selected background characteristics, National Family Health Survey, 2015-16

\begin{tabular}{|c|c|c|c|c|c|c|}
\hline \multirow[b]{2}{*}{ Background characteristics } & \multicolumn{3}{|c|}{ IFA100 } & \multicolumn{3}{|c|}{ SF } \\
\hline & $\%$ & $95 \% \mathrm{Cl}$ & $P$ & $\%$ & $95 \% \mathrm{Cl}$ & $P$ \\
\hline Age at childbirth & \multicolumn{3}{|c|}{$\chi^{2}(5)=473.5$} & \multicolumn{3}{|c|}{$\chi^{2}(5)=1604 \cdot 8$} \\
\hline$\leq 19$ & 27.5 & $26 \cdot 2,28 \cdot 7$ & $<0.001$ & $59 \cdot 3$ & $58 \cdot 0,60.5$ & $<0.001$ \\
\hline $20-24$ & $30 \cdot 8$ & $30 \cdot 2,31 \cdot 4$ & & $56 \cdot 0$ & $55 \cdot 4,56 \cdot 6$ & \\
\hline $25-29$ & 31.9 & $31 \cdot 2,32 \cdot 6$ & & $50 \cdot 1$ & $49 \cdot 4,50 \cdot 7$ & \\
\hline $30-34$ & 29.6 & $28 \cdot 6,30 \cdot 5$ & & $46 \cdot 4$ & $45 \cdot 4,47 \cdot 3$ & \\
\hline $35-39$ & $24 \cdot 1$ & $22 \cdot 5,25 \cdot 8$ & & 43.3 & $41 \cdot 7,44 \cdot 8$ & \\
\hline $40-49$ & $17 \cdot 2$ & $15 \cdot 3,19 \cdot 4$ & & 39.5 & $37 \cdot 1,41.9$ & \\
\hline Women's education & \multicolumn{3}{|c|}{$\chi^{2}(5)=1210 \cdot 2$} & \multicolumn{3}{|c|}{$\chi^{2}(5)=4210 \cdot 8$} \\
\hline No schooling & $15 \cdot 7$ & $15 \cdot 2,16 \cdot 2$ & $<0.001$ & $50 \cdot 1$ & $49.4,50.8$ & $<0.001$ \\
\hline$<5$ years & 23.8 & $22 \cdot 5,25 \cdot 2$ & & $61 \cdot 8$ & $60 \cdot 3,63 \cdot 2$ & \\
\hline $5-7$ years & $28 \cdot 0$ & $27 \cdot 1,29 \cdot 0$ & & $60 \cdot 1$ & $59 \cdot 2,61 \cdot 0$ & \\
\hline 8-9 years & $30 \cdot 4$ & $29 \cdot 6,31 \cdot 3$ & & 58.7 & $57 \cdot 9,59 \cdot 6$ & \\
\hline $10-11$ years & $40 \cdot 8$ & $39.7,41.9$ & & 55.7 & $54.6,56 \cdot 8$ & \\
\hline$\geq 12$ years & $46 \cdot 7$ & $45 \cdot 8,47 \cdot 7$ & & $40 \cdot 3$ & $39 \cdot 4,41 \cdot 2$ & \\
\hline Parity & \multicolumn{3}{|c|}{$\chi^{2}(3)=4565 \cdot 2$} & \multicolumn{3}{|c|}{$\chi^{2}(3)=521 \cdot 7$} \\
\hline 1 & $36 \cdot 0$ & $35 \cdot 3,36 \cdot 7$ & $<0.001$ & $52 \cdot 0$ & $51 \cdot 3,52 \cdot 7$ & $<0.001$ \\
\hline $2-3$ & 31.3 & $30 \cdot 7,31 \cdot 9$ & & $54 \cdot 2$ & $53 \cdot 6,54 \cdot 8$ & \\
\hline $4-5$ & $16 \cdot 0$ & $15 \cdot 3,16 \cdot 8$ & & $49 \cdot 3$ & $48 \cdot 4,50 \cdot 3$ & \\
\hline$\geq 6$ & $10 \cdot 1$ & $9 \cdot 2,11 \cdot 0$ & & $42 \cdot 2$ & $40 \cdot 7,43 \cdot 7$ & \\
\hline Gender composition of living children & \multicolumn{3}{|c|}{$\chi^{2}(1)=482 \cdot 5$} & \multicolumn{3}{|c|}{$\chi^{2}(1)=74.8$} \\
\hline No sons & $34 \cdot 0$ & $33 \cdot 3,34.7$ & $<0.001$ & $54 \cdot 0$ & $53 \cdot 3,54 \cdot 8$ & $<0.001$ \\
\hline At least one son & 28.9 & $28 \cdot 4,29 \cdot 4$ & & $51 \cdot 8$ & $51 \cdot 3,52 \cdot 3$ & \\
\hline Prenatal care timing (months of pregnancy) & \multicolumn{3}{|c|}{$\begin{array}{l}28 \cdot 4,29 \cdot 4 \\
\chi^{2}(3)=9233 \cdot 0\end{array}$} & & $\chi^{2}(3)=4526.5$ & \\
\hline No antenatal care & $10 \cdot 7$ & $10 \cdot 0,11.4$ & $<0.001$ & $35 \cdot 7$ & $34.8,36.6$ & $<0.001$ \\
\hline Early $\leq 3$ months & 37.7 & $37 \cdot 1,38 \cdot 3$ & & $56 \cdot 0$ & $55 \cdot 5,56 \cdot 6$ & \\
\hline Intermediate $4-5$ months & $25 \cdot 1$ & $24 \cdot 3,25 \cdot 9$ & & $57 \cdot 2$ & $56 \cdot 4,58 \cdot 1$ & \\
\hline Late $\geq 6$ months & $29 \cdot 0$ & $27 \cdot 4,30 \cdot 6$ & & $50 \cdot 3$ & $48 \cdot 7,51.9$ & \\
\hline Total prenatal visits & & $\chi^{2}(1)=1$ & & & $\chi^{2}(1)=1722 \cdot 7$ & \\
\hline$<4$ & $16 \cdot 5$ & $16 \cdot 1,17 \cdot 0$ & $<0.001$ & $47 \cdot 6$ & $47.0,48 \cdot 1$ & $<0.001$ \\
\hline$\geq 4$ & 43.5 & $42 \cdot 8,44 \cdot 1$ & & $57 \cdot 1$ & $56 \cdot 4,57 \cdot 7$ & \\
\hline Met healthcare providers during pregnancy & & $\chi^{2}(1)=159$ & & & $\chi^{2}(4)=1 \cdot 10 \cdot 4$ & \\
\hline Not met & $26 \cdot 4$ & $25 \cdot 8,27 \cdot 1$ & $<0.001$ & $31 \cdot 1$ & $30.5,31.6$ & $<0.001$ \\
\hline Met at home only & 31.3 & $30 \cdot 4,32 \cdot 2$ & & 71.5 & $70 \cdot 7,72 \cdot 2$ & \\
\hline Met elsewhere & $35 \cdot 8$ & $35 \cdot 0,36 \cdot 6$ & & $72 \cdot 6$ & $71.9,73.4$ & \\
\hline Met at both places & $36 \cdot 0$ & $34 \cdot 7,37 \cdot 4$ & & 81.9 & $81 \cdot 0,82 \cdot 7$ & \\
\hline ANC provider & & $\chi^{2}(1)=874$ & & & $\chi^{2}(3)=8102 \cdot 6$ & \\
\hline Others & 23.2 & $20 \cdot 5,26 \cdot 2$ & $<0.001$ & 54 & $50.5,57.5$ & $<0.001$ \\
\hline Doctor & 39.8 & $38 \cdot 9,40 \cdot 7$ & & 46.5 & $45 \cdot 7,47 \cdot 3$ & \\
\hline ANM/nurse/midwife/LHV & $31 \cdot 0$ & $30 \cdot 4,31.6$ & & 56.5 & $55 \cdot 8,57 \cdot 2$ & \\
\hline CHW/ICDS/ASHA & $30 \cdot 0$ & $29 \cdot 1,31 \cdot 0$ & & $76 \cdot 4$ & $75 \cdot 6,77 \cdot 2$ & \\
\hline No ANC & $10 \cdot 3$ & $9.6,11.0$ & & 35.6 & $34 \cdot 7,36 \cdot 5$ & \\
\hline Place where ANC received & & $\chi^{2}(1)=111$ & & & $x^{2}(3)=3 \cdot 60$ & \\
\hline At home only & 24.5 & $23 \cdot 2,25.9$ & $<0.001$ & $48 \cdot 6$ & $47 \cdot 1,50 \cdot 2$ & $<0.001$ \\
\hline Government hospital only & $40 \cdot 1$ & $38 \cdot 8,41 \cdot 4$ & & $51 \cdot 8$ & $50 \cdot 5,53 \cdot 1$ & \\
\hline $\mathrm{CHC} /$ rural hospital/block PHC only & $24 \cdot 1$ & $23 \cdot 1,25 \cdot 2$ & & 60.5 & $59 \cdot 2,61 \cdot 7$ & \\
\hline PHC only & 33.4 & $31 \cdot 7,35 \cdot 1$ & & 63.7 & $62 \cdot 0,65 \cdot 4$ & \\
\hline ICDS/SC only & $27 \cdot 4$ & $25 \cdot 3,29 \cdot 7$ & & $64 \cdot 4$ & $62 \cdot 1,66 \cdot 6$ & \\
\hline Private hospital/clinic only & $40 \cdot 4$ & $39 \cdot 4,41.5$ & & $40 \cdot 3$ & $39 \cdot 4,41 \cdot 2$ & \\
\hline At least government hospital & $44 \cdot 9$ & $42 \cdot 5,47 \cdot 3$ & & $52 \cdot 7$ & $50 \cdot 3,55 \cdot 0$ & \\
\hline At least $\mathrm{CHC} /$ rural hospital/block $\mathrm{PHC}$ & $35 \cdot 8$ & $32 \cdot 1,39 \cdot 8$ & & $65 \cdot 3$ & $61 \cdot 4,69 \cdot 1$ & \\
\hline At least PHC & $45 \cdot 2$ & $41 \cdot 2,49 \cdot 3$ & & $68 \cdot 1$ & $64 \cdot 3,71 \cdot 8$ & \\
\hline At least ICDS/SC & $26 \cdot 0$ & $25 \cdot 1,26 \cdot 9$ & & $76 \cdot 7$ & $75 \cdot 9,77.5$ & \\
\hline Private hospital/clinic & 37.4 & $36 \cdot 3,38 \cdot 6$ & & $56 \cdot 7$ & $55 \cdot 5,57 \cdot 9$ & \\
\hline No ANC & $10 \cdot 3$ & $9 \cdot 6,11 \cdot 0$ & & $35 \cdot 6$ & $34 \cdot 7,36.5$ & \\
\hline Wealth quintile & & $\chi^{2}(4)=1$ & & & $\chi^{2}(4)=7653.6$ & \\
\hline Poorest & $14 \cdot 4$ & $13.9,15 \cdot 0$ & $<0.001$ & 54.5 & $53 \cdot 7,55 \cdot 2$ & $<0.001$ \\
\hline Poorer & 23.2 & $22 \cdot 5,23 \cdot 8$ & & $61 \cdot 3$ & $60 \cdot 5,62 \cdot 0$ & \\
\hline Middle & $32 \cdot 8$ & $31.9,33 \cdot 6$ & & $60 \cdot 0$ & $59 \cdot 1,60 \cdot 8$ & \\
\hline Richer & 39.6 & $38.5,40 \cdot 6$ & & $50 \cdot 2$ & $49 \cdot 2,51 \cdot 1$ & \\
\hline Richest & 48.2 & $47 \cdot 1,49 \cdot 4$ & & 31.9 & $30.8,32.9$ & \\
\hline Caste & & $\chi^{2}(3)=530$ & & & $\chi^{2}(3)=3889.2$ & \\
\hline Others & $34 \cdot 8$ & $33 \cdot 7,35 \cdot 8$ & $<0.001$ & $42 \cdot 3$ & $41 \cdot 3,43.4$ & $<0.001$ \\
\hline Scheduled Castes & $28 \cdot 6$ & $27 \cdot 6,29 \cdot 6$ & & $59 \cdot 1$ & $58 \cdot 2,60 \cdot 1$ & \\
\hline Scheduled Tribes & $26 \cdot 8$ & $25 \cdot 7,27 \cdot 9$ & & $66 \cdot 3$ & $65 \cdot 1,67 \cdot 5$ & \\
\hline Other Backward Classes & 30.2 & $29 \cdot 7,30 \cdot 8$ & & 50.9 & $50 \cdot 3,51 \cdot 6$ & \\
\hline
\end{tabular}


Table 1 Continued

\begin{tabular}{|c|c|c|c|c|c|c|}
\hline \multirow[b]{2}{*}{ Background characteristics } & \multicolumn{3}{|c|}{ IFA100 } & \multicolumn{3}{|c|}{ SF } \\
\hline & $\%$ & $95 \% \mathrm{Cl}$ & $P$ & $\%$ & $95 \% \mathrm{Cl}$ & $P$ \\
\hline Religion & \multicolumn{3}{|c|}{$\chi^{2}(2)=1035 \cdot 4$} & \multicolumn{3}{|c|}{$\chi^{2}(2)=1645.5$} \\
\hline Hindu & $30 \cdot 8$ & $30 \cdot 3,31 \cdot 3$ & $<0.001$ & 54.4 & $53.9,54.9$ & $<0.001$ \\
\hline Muslim & $24 \cdot 7$ & $23 \cdot 6,25 \cdot 7$ & & 41.9 & $40 \cdot 6,43 \cdot 2$ & \\
\hline Others & 41.4 & $39 \cdot 3,43.5$ & & $56 \cdot 2$ & $54 \cdot 2,58 \cdot 1$ & \\
\hline Place of residence & \multicolumn{3}{|c|}{$\chi^{2}(1)=4236 \cdot 4$} & \multicolumn{3}{|c|}{$\chi^{2}(1)=8128 \cdot 7$} \\
\hline Rural & $25 \cdot 9$ & $25 \cdot 5,26 \cdot 3$ & $<0.001$ & $59 \cdot 1$ & $58 \cdot 7,59 \cdot 6$ & $<0.001$ \\
\hline Urban & $40 \cdot 8$ & $39 \cdot 7,42 \cdot 0$ & & $36 \cdot 6$ & $35 \cdot 6,37 \cdot 6$ & \\
\hline Region & & $\chi^{2}(5)=1$ & & & $x^{2}(5)=3377$ & \\
\hline South & $56 \cdot 8$ & $55 \cdot 6,58 \cdot 0$ & $<0.001$ & $61 \cdot 8$ & $60 \cdot 6,62 \cdot 9$ & $<0.001$ \\
\hline North & $29 \cdot 4$ & $28 \cdot 0,30 \cdot 9$ & & $42 \cdot 0$ & $41 \cdot 0,43 \cdot 0$ & \\
\hline Central & $17 \cdot 3$ & $16 \cdot 8,17 \cdot 7$ & & $49 \cdot 2$ & $48 \cdot 5,50 \cdot 0$ & \\
\hline East & $19 \cdot 6$ & $19 \cdot 0,20 \cdot 3$ & & $57 \cdot 3$ & $56 \cdot 4,58 \cdot 2$ & \\
\hline Northeast & $30 \cdot 5$ & $29 \cdot 4,31 \cdot 7$ & & $53 \cdot 1$ & $51 \cdot 7,54 \cdot 5$ & \\
\hline West & 39.5 & $37 \cdot 8,41 \cdot 2$ & & $46 \cdot 4$ & $44.8,47.9$ & \\
\hline Total & $30 \cdot 3$ & $29 \cdot 9,30 \cdot 8$ & & $52 \cdot 4$ & $52 \cdot 0,52 \cdot 9$ & \\
\hline
\end{tabular}

ICDS, Integrated Child Development Services; SC, sub-centre; ANC, Antenatal Care; ANM, Auxilliary Nurse Midwife; LHV, Lady Health Visitor; CHW, Community Health Worker; ICDS, Integrated Child Development Services; ASHA, Accredited Social Health Activist; CHC, Community Health Centre; PHC, Primary Health Centre.

compared to those from the NHFS. Similarly, women from the community with more female illiteracy had $14 \%$ less probability of using IFA100. Similarly, the likelihood of IFA100 use was higher among those districts with a higher rate of pregnancy registration (OR $12.30 .95 \%$ CI $7.94,19.06$ ).

In the case of women receiving SF (Table 4), findings suggest that women's age at childbirth, educational status, parity, economic status, caste and religious affiliation were significantly associated factors. Those who registered their pregnancy in the first trimester (OR 1.41, 95\% CI 1.16, 1.72), who had four or more ANC visits (OR 1.08, $95 \%$ CI 1.05, 1.12) and who had at least one ANC visit at an ICDS centre or sub-centre (OR 1.97, $95 \%$ CI 1.85, 2.10) showed higher odds of receiving SF compared to women receiving no ANC and who received ANC at home. Similarly, women's contact with healthcare providers during pregnancy increased their chance of receiving SF by nearly five times compared to those not meeting healthcare providers. The likelihood of women receiving SF was nearly $87 \%$ higher in the HFS than in the NHFS. Further, in communities where more than three-fourths of women were illiterate, there was an $18 \%$ less probability of their receiving $\mathrm{SF}$. In addition, a higher proportion of pregnancies registered in a district confirmed a higher probability of women in that district receiving SF.

\section{Comparison between High Focus States and non-High Focus States}

Findings suggest that caste affiliation significantly determined the consumption of IFA100 in HFS but not in the case of NHFS (see online supplementary material, Supplemental Table S2). Further, women from NHFS who visited government healthcare facilities for ANC were more likely to consume IFA than those who received ANC at home. For districts with $>75 \%$ coverage of pregnancy registration, IFA use was nearly 29 and 5.6 times higher in NHFS and HFS, respectively, compared to districts with $<25 \%$ pregnancy registration.

On the other hand, women who received ANC at any government health facility in NHFS had higher odds of receiving SF compared to those who received ANC at home (see online supplementary material, Supplemental Table S3). However, in the case of HFS, there was no significant difference in SF receipt between women who used a government health facility for ANC and those who did not. Further, in the case of HFS, the association between women residing in a poor community and receiving SF was statistically significant, which was not true in the context of NHFS.

\section{Administrative area-specific (district) contextual effects}

The random intercept-only model (Table 2) applied for IFA consumption illustrated that there were nearly 26 and $44 \%$ variances (out of total variances) in the use of IFA at district and community levels, respectively, which could be due to some unobserved factors at these levels. However, the full IFA model (Table 3 ) explains around $62 \%$ of district-level variances, illustrating the observed differences in the proportion of pregnancies registered across the districts. The final IFA model suggests that there were about 10 and $30 \%$ of variances in the use of IFA, which could be explained through the differences in elements at the district and community levels, respectively.

A district-level residual map for IFA (Fig. 1(b)) clearly shows the clusters of districts in western Rajasthan, parts of Jharkhand, Maharashtra, Arunachal Pradesh, Nagaland and Tripura, which had relatively higher negative variances, suggesting that, due to some unobserved district-level factors, the use of IFA was at a suboptimal level in these clusters of districts. Similarly, around 20 and $38 \%$ of 


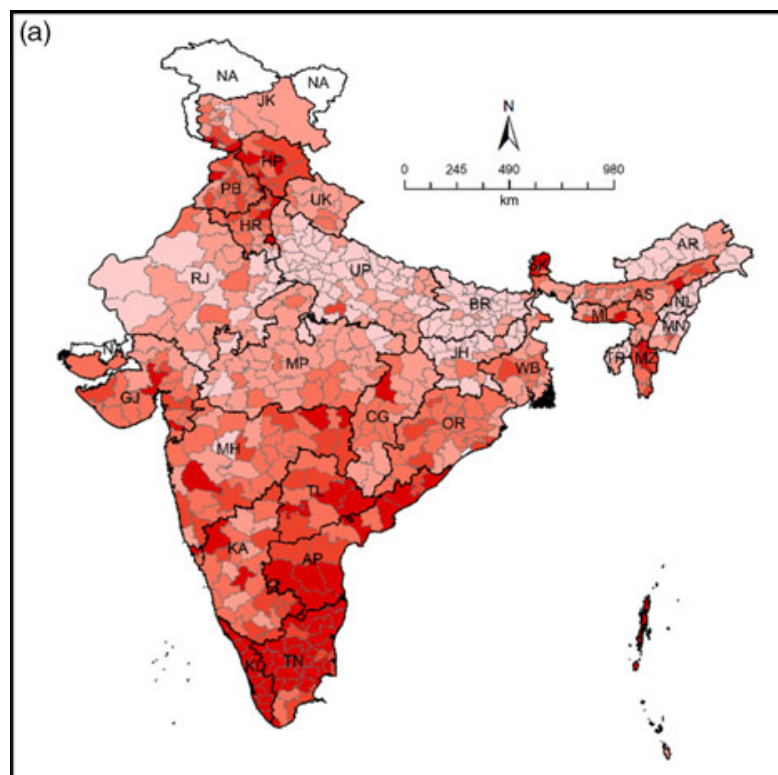

(c)

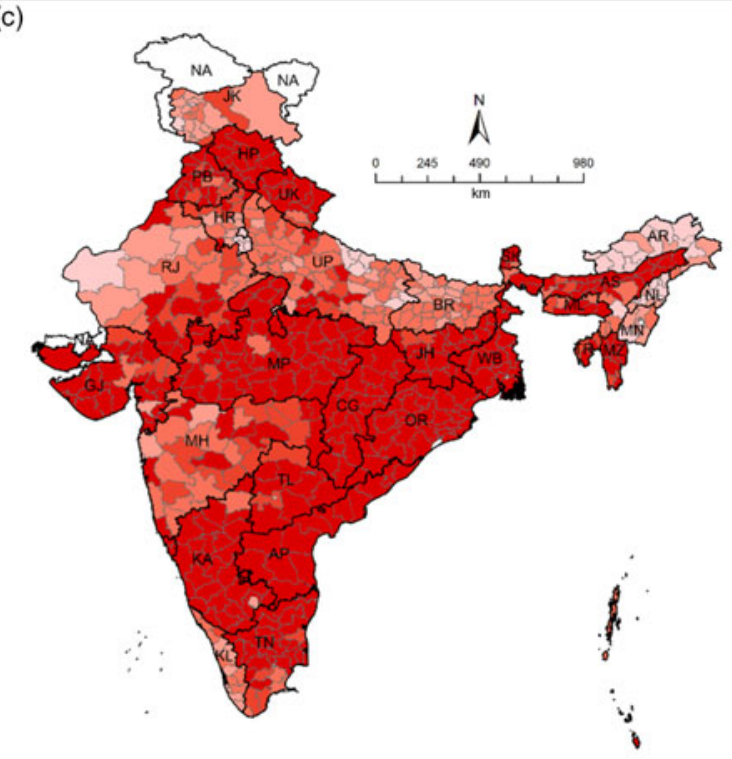

(b)

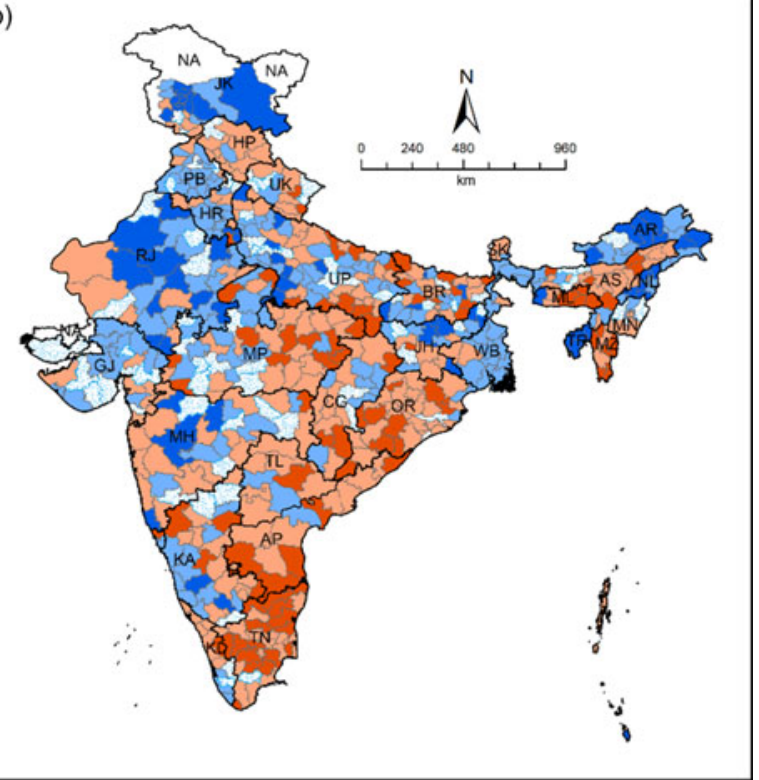

(d)

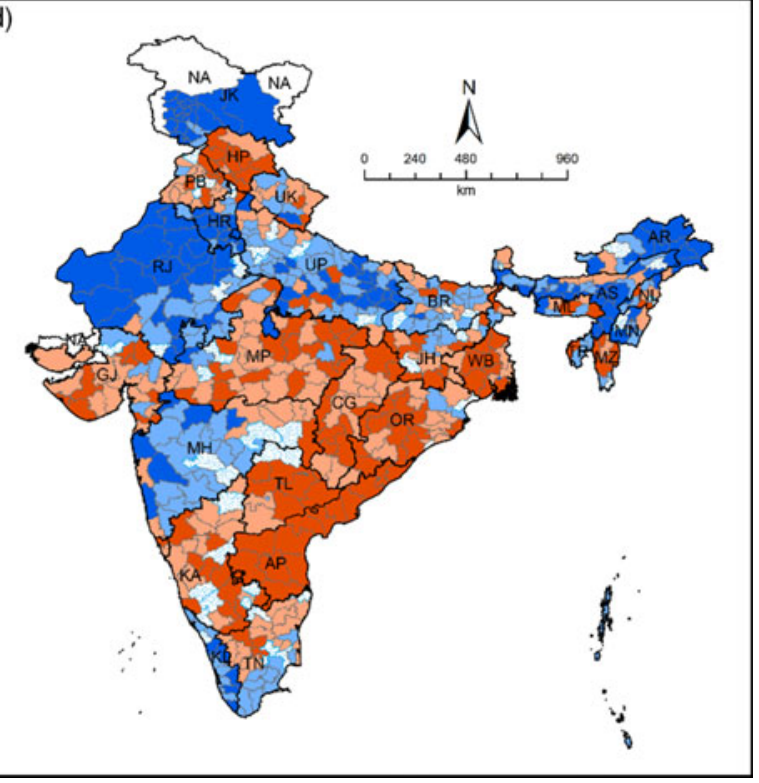

Fig. 1 Iron and folic acid tablets/syrup consumption (\%) for at least $100 \mathrm{~d}$ (IFA100), supplementary food (SF) received (\%) and their model-based heterogeneity across districts of India, National Family Health Survey-4 (2015-16). (a) Proportion of women (15-49) (\%) consuming IFA100 during pregnancy. (b) District-level residuals (variances) map for IFA100. (c) Proportion of mothers (\%) receiving supplementary food from an anganwadi/Integrated Child Development Services centre during pregnancy. (d) District-level residuals (variances) map for SF. (a and c) , <15.0; , 15.0-30.0; , 30.1-45.0; , 45.1-55.0; , > 55.0; 스, data not available. (b and d) $\square,<-0.70$; $\square,-0.70$ to $-0.11 ; \square,-0.1$ to $0.10 ; \square, 0.11-0.70 ; \square,<0.70$; $\square$, data not available

Table 2 Parameter coefficients for the multilevel model (random intercept-only model, without covariates) for iron and folic acid tablets/syrup for at least $100 \mathrm{~d}$ (IFA100) and supplementary food (SF), National Family Health Survey (2015-16)

\begin{tabular}{|c|c|c|c|c|c|c|}
\hline \multirow[b]{2}{*}{ Random effect } & \multicolumn{3}{|c|}{ IFA100 } & \multicolumn{3}{|c|}{ SF } \\
\hline & $s^{2}$ & $95 \% \mathrm{Cl}$ & SE & $s^{2}$ & $95 \% \mathrm{Cl}$ & SE \\
\hline \multicolumn{7}{|c|}{ Random-effects parameters } \\
\hline District & 1.525 & $1.357,1.713$ & 0.091 & $2 \cdot 152$ & $1.918,2.415$ & 0.126 \\
\hline PSU & 1.043 & $0.999,1.088$ & 0.023 & 1.497 & $1.444,1.553$ & 0.028 \\
\hline \multicolumn{7}{|c|}{ Intraclass correlation coefficient } \\
\hline District & 0.260 & $0.239,0.283$ & 0.011 & 0.310 & $0.286,0.335$ & 0.013 \\
\hline PSU & 0.438 & $0.421,0.456$ & 0.009 & 0.526 & $0.508,0.544$ & 0.009 \\
\hline
\end{tabular}

$s^{2}$, variance; PSU, primary sampling unit. 


\begin{tabular}{|c|c|c|c|c|c|c|c|c|c|c|c|c|}
\hline \multirow[b]{2}{*}{ Background variables } & \multicolumn{4}{|c|}{ Model 1 (individual/household factors) } & \multicolumn{4}{|c|}{ Model 2 (model $1+$ programme factors) } & \multicolumn{4}{|c|}{$\begin{array}{c}\text { Model } 3 \text { (model } 2+\text { compositional/ } \\
\text { contextual factors) }\end{array}$} \\
\hline & OR & SE & $95 \% \mathrm{Cl}$ & $P$ & OR & SE & $95 \% \mathrm{Cl}$ & $P$ & OR & SE & $95 \% \mathrm{Cl}$ & $P$ \\
\hline \multicolumn{13}{|l|}{ Fixed effect } \\
\hline \multicolumn{13}{|l|}{ Individual/household factors } \\
\hline \multicolumn{13}{|l|}{ Age at childbirth } \\
\hline$\leq 19$ (ref.) & 1.00 & & & & 1.00 & & & & 1.00 & & & \\
\hline $20-24$ & $1 \cdot 10$ & & $1.04,1.15$ & 0.001 & 1.07 & & $1.02,1.13$ & 0.010 & 1.07 & & $1.02,1.13$ & 0.011 \\
\hline $25-29$ & 1.24 & & $1.17,1.31$ & $<0.001$ & 1.20 & & $1 \cdot 14,1 \cdot 27$ & $<0.001$ & 1.20 & & $1 \cdot 13,1.27$ & $<0.001$ \\
\hline $30-34$ & 1.28 & & $1.20,1.37$ & $<0.001$ & $1 \cdot 24$ & & $1.16,1.32$ & $<0.001$ & 1.23 & & $1.15,1.31$ & $<0.001$ \\
\hline $35-39$ & 1.30 & & $1.20,1.42$ & $<0.001$ & 1.27 & & $1.17,1.38$ & $<0.001$ & 1.26 & & $1.16,1.37$ & $<0.001$ \\
\hline $40-49$ & 1.23 & & $1.08,1.41$ & 0.002 & 1.26 & & $1.10,1.44$ & 0.001 & 1.25 & & $1.09,1.43$ & 0.002 \\
\hline \multicolumn{13}{|l|}{ Women's education } \\
\hline No schooling (ref.) & 1.00 & & & & 1.00 & & & & 1.00 & & & \\
\hline$<5$ years & $1 \cdot 12$ & & $1.05,1.19$ & $<0.001$ & 1.06 & & $1.00,1.13$ & 0.058 & 1.05 & & $0.99,1.12$ & 0.112 \\
\hline $5-7$ years & 1.24 & & $1 \cdot 19,1.30$ & $<0.001$ & $1 \cdot 15$ & & $1 \cdot 10,1 \cdot 21$ & $<0.001$ & 1.13 & & $1.08,1.19$ & $<0.001$ \\
\hline $8-9$ years & 1.35 & & $1.29,1.41$ & $<0.001$ & 1.23 & & $1 \cdot 17,1 \cdot 28$ & $<0.001$ & 1.20 & & $1 \cdot 15,1.26$ & $<0.001$ \\
\hline $10-11$ years & 1.59 & & $1.51,1.68$ & $<0.001$ & 1.43 & & $1.36,1.51$ & $<0.001$ & 1.40 & & $1.33,1.47$ & $<0.001$ \\
\hline$\geq 12$ years & 2.04 & & $1.94,2 \cdot 14$ & $<0.001$ & 1.78 & & $1.69,1.87$ & $<0.001$ & 1.75 & & $1.66,1.84$ & $<0.001$ \\
\hline \multicolumn{13}{|l|}{ Parity } \\
\hline 1 (ref.) & 1.00 & & & & 1.00 & & & & 1.00 & & & \\
\hline $2-3$ & 0.87 & & $0.84,0.90$ & $<0.001$ & 0.90 & & $0.87,0.93$ & $<0.001$ & 0.90 & & $0.87,0.93$ & $<0.001$ \\
\hline $4-5$ & 0.68 & & $0.64,0.72$ & $<0.001$ & 0.74 & & $0.71,0.79$ & $<0.001$ & 0.75 & & $0.71,0.79$ & $<0.001$ \\
\hline$\geq 6$ & 0.58 & & $0.53,0.64$ & $<0.001$ & 0.67 & & $0.61,0.74$ & $<0.001$ & 0.68 & & $0.62,0.75$ & $<0.001$ \\
\hline \multicolumn{13}{|c|}{ Gender composition of living children } \\
\hline No sons (ref.) & 1.00 & & & & 1.00 & & & & 1.00 & & & \\
\hline At least one son & 0.97 & & $0.95,1.00$ & 0.070 & 0.98 & & $0.95,1.01$ & 0.133 & 0.98 & & $0.95,1.01$ & 0.138 \\
\hline \multicolumn{13}{|l|}{ Wealth quintile } \\
\hline Poorest (ref.) & 1.00 & & & & 1.00 & & & & 1.00 & & & \\
\hline Poorer & 1.22 & & $1 \cdot 17,1 \cdot 28$ & $<0.001$ & 1.14 & & $1.08,1.19$ & $<0.001$ & 1.11 & & $1.06,1 \cdot 16$ & $<0.001$ \\
\hline Middle & 1.43 & & $1.36,1.50$ & $<0.001$ & $1 \cdot 28$ & & $1.22,1.35$ & $<0.001$ & 1.23 & & $1.16,1.29$ & $<0.001$ \\
\hline Richer & 1.66 & & $1.58,1.76$ & $<0.001$ & 1.45 & & $1.37,1.53$ & $<0.001$ & 1.37 & & $1.29,1.45$ & $<0.001$ \\
\hline Richest & $2 \cdot 19$ & & $2 \cdot 06,2 \cdot 33$ & $<0.001$ & 1.86 & & $1.74,1.98$ & $<0.001$ & 1.74 & & $1.62,1.86$ & $<0.001$ \\
\hline \multicolumn{13}{|l|}{ Caste } \\
\hline Others (ref.) & 1.00 & & & & 1.00 & & & & 1.00 & & & \\
\hline Scheduled Castes & 0.88 & & $0.84,0.92$ & $<0.001$ & 0.88 & & $0.84,0.93$ & $<0.001$ & 0.88 & & $0.84,0.93$ & $<0.001$ \\
\hline Scheduled Tribes & 0.89 & & $0.84,0.95$ & $<0.001$ & 0.92 & & $0.87,0.97$ & 0.004 & 0.93 & & $0.88,0.99$ & 0.016 \\
\hline Other Backward Classes & 0.91 & & $0.87,0.95$ & $<0.001$ & 0.92 & & $0.88,0.96$ & $<0.001$ & 0.92 & & $0.89,0.96$ & $<0.001$ \\
\hline \multicolumn{13}{|l|}{ Religion } \\
\hline Hindu (ref.) & 1.00 & & & & 1.00 & & & & 1.00 & & & \\
\hline Muslim & 0.85 & & $0.81,0.90$ & $<0.001$ & 0.86 & & $0.82,0.91$ & $<0.001$ & 0.86 & & $0.81,0.90$ & $<0.001$ \\
\hline Others & 0.98 & & $0.92,1.04$ & 0.510 & 0.98 & & $0.92,1.05$ & 0.583 & 0.98 & & $0.91,1.04$ & 0.447 \\
\hline \multicolumn{13}{|c|}{ Programme factors } \\
\hline \multicolumn{13}{|c|}{ Prenatal care timing (months of pregnancy) } \\
\hline No ANC (ref.) & & & & & 1.00 & & & & 1.00 & & & \\
\hline Early ( $\leq 3$ months) & & & & & 1.33 & & $1.09,1.62$ & 0.004 & 1.33 & & $1.09,1.62$ & 0.004 \\
\hline Intermediate (4-5 months) & & & & & 1.14 & & $0.94,1.40$ & 0.186 & 1.15 & & $0.94,1.40$ & 0.167 \\
\hline Late ( $\geq 6$ months) & & & & & 1.06 & & $0.86,1.30$ & 0.578 & 1.06 & & $0.87,1.30$ & 0.549 \\
\hline
\end{tabular}




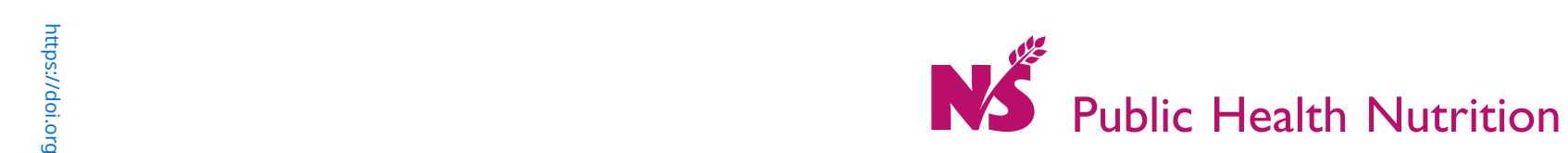

Table 3 Continued

\begin{tabular}{|c|c|c|c|c|c|c|c|c|c|c|c|c|}
\hline \multirow[b]{2}{*}{ Background variables } & \multicolumn{4}{|c|}{ Model 1 (individual/household factors) } & \multicolumn{4}{|c|}{ Model 2 (model $1+$ programme factors) } & \multicolumn{4}{|c|}{$\begin{array}{c}\text { Model } 3 \text { (model } 2+\text { compositional/ } \\
\text { contextual factors) }\end{array}$} \\
\hline & OR & SE & $95 \% \mathrm{Cl}$ & $P$ & OR & SE & $95 \% \mathrm{Cl}$ & $P$ & OR & SE & $95 \% \mathrm{Cl}$ & $P$ \\
\hline \multicolumn{13}{|l|}{ Total prenatal visits } \\
\hline$<4$ (ref.) & & & & & 1.00 & & & & 1.00 & & & \\
\hline$\geq 4$ & & & & & 1.76 & & $1.70,1.82$ & $<0.001$ & 1.74 & & $1.68,1.79$ & $<0.001$ \\
\hline \multicolumn{13}{|l|}{ Met healthcare providers during pregnancy } \\
\hline Not met (ref.) & & & & & 1.00 & & & & 1.00 & & & \\
\hline Met at home only & & & & & 1.25 & & $1.21,1.30$ & $<0.001$ & 1.25 & & $1.21,1.30$ & $<0.001$ \\
\hline Met elsewhere only & & & & & 1.26 & & $1.22,1.31$ & $<0.001$ & 1.26 & & $1.22,1.31$ & $<0.001$ \\
\hline Met at both places & & & & & 1.29 & & $1.23,1.35$ & $<0.001$ & 1.29 & & $1.23,1.35$ & $<0.001$ \\
\hline \multicolumn{13}{|l|}{ Place where ANC received } \\
\hline At home only (ref.) & & & & & 1.00 & & & & 1.00 & & & \\
\hline Government hospital only & & & & & 1.24 & & $1.16,1.32$ & $<0.001$ & 1.22 & & $1.14,1.3$ & $<0.001$ \\
\hline $\mathrm{CHC} /$ rural hospital/block PHC only & & & & & 1.12 & & $1.04,1.20$ & 0.002 & 1.12 & & $1.04,1.20$ & 0.002 \\
\hline PHC only & & & & & 1.31 & & $1.21,1.42$ & $<0.001$ & 1.31 & & $1.21,1.42$ & $<0.001$ \\
\hline ICDS/SC only & & & & & 1.07 & & $0.97,1.18$ & 0.164 & 1.07 & & $0.97,1.17$ & 0.181 \\
\hline Private hospital/clinic only & & & & & 1.31 & & $1.23,1.40$ & $<0.001$ & 1.31 & & $1.23,1.40$ & $<0.001$ \\
\hline At least government hospital & & & & & 1.34 & & $1.23,1.45$ & $<0.001$ & 1.32 & & $1.22,1.43$ & $<0.001$ \\
\hline At least $\mathrm{CHC} /$ rural hospital/block PHC & & & & & 1.36 & & $1.19,1.56$ & $<0.001$ & 1.35 & & $1.18,1.55$ & $<0.001$ \\
\hline At least PHC & & & & & 1.36 & & $1.19,1.56$ & $<0.001$ & 1.35 & & $1.18,1.55$ & $<0.001$ \\
\hline At least ICDS/SC & & & & & 1.20 & & $1 \cdot 12,1 \cdot 28$ & $<0.001$ & $1 \cdot 20$ & & $1 \cdot 12,1 \cdot 28$ & $<0.001$ \\
\hline Private hospital/clinic & & & & & 1.21 & & $1.13,1.30$ & $<0.001$ & 1.21 & & $1.13,1.29$ & $<0.001$ \\
\hline No ANC & & & & & 0.83 & & $0.67,1.02$ & 0.069 & 0.84 & & $0.68,1.03$ & 0.089 \\
\hline \multicolumn{13}{|l|}{ Compositional/contextual factors } \\
\hline \multicolumn{13}{|l|}{ Proportion of illiterate women in PSU (\%) } \\
\hline $0-25$ (ref.) & & & & & & & & & 1.00 & & & \\
\hline $25-50$ & & & & & & & & & 1.00 & & $0.95,1.05$ & 0.980 \\
\hline $50-75$ & & & & & & & & & 0.96 & & $0.90,1.01$ & 0.132 \\
\hline $75-100$ & & & & & & & & & 0.86 & & $0.80,0.92$ & 0.000 \\
\hline \multicolumn{13}{|l|}{ Proportion of poor women in PSU (\%) } \\
\hline $0-25$ (ref.) & & & & & & & & & 1.00 & & & \\
\hline $25-50$ & & & & & & & & & 0.98 & & $0.92,1.04$ & 0.448 \\
\hline $50-75$ & & & & & & & & & 0.98 & & $0.92,1.05$ & 0.561 \\
\hline $75-100$ & & & & & & & & & 0.94 & & $0.87,1.02$ & 0.140 \\
\hline \multicolumn{13}{|l|}{ Pregnancies registered in the district (\%) } \\
\hline $0-25$ (ref.) & & & & & & & & & 1.00 & & & \\
\hline $25-50$ & & & & & & & & & $3 \cdot 10$ & & $2.08,4.62$ & $<0.001$ \\
\hline $50-75$ & & & & & & & & & $7 \cdot 26$ & & $4.92,10.71$ & $<0.001$ \\
\hline $75-100$ & & & & & & & & & $12 \cdot 30$ & & $7.94,19.06$ & $<0.001$ \\
\hline \multicolumn{13}{|l|}{ Place of current residence } \\
\hline Rural (ref.) & & & & & & & & & 1.00 & & & \\
\hline Urban & & & & & & & & & 1.01 & & $0.96,1.06$ & 0.697 \\
\hline \multicolumn{13}{|l|}{ Region } \\
\hline Non-High Focus States (ref.) & & & & & & & & & 1.00 & & & \\
\hline High Focus States & & & & & & & & & 0.69 & & $0.61,0.79$ & $<0.001$ \\
\hline
\end{tabular}


variances in SF receipt could be explained through the differences in elements at the district and community levels, respectively (Table 4). A district-level residual map for SF use (Fig. 1(d)) shows clusters of districts in Rajasthan, Haryana, eastern Uttar Pradesh, parts of Maharashtra, Kerala, Assam, Arunachal Pradesh, Manipur, and Jammu and Kashmir having relatively higher negative variances, suggesting that, due to some unobserved district-level factors, the use of SF was at a suboptimal level in these clusters of districts.

\section{Discussion}

The findings of this study demonstrate that the determinants of utilisation of two most essential interventions of maternal nutrition during pregnancy include a few important components of prenatal healthcare programmes and aggregated (community-level) compositional or contextual factors.

\section{Socioeconomic factors}

The findings indicated an increasing trend of utilisation of IFA with increasing age and level of education, which is well supported by other studies ${ }^{(34,35)}$. However, older women were found to be less likely to receive SF. Education status of women has been recorded in recent literature to factor in the overall awareness about relevant reproductive health programmes and beneficial policies at their disposal ${ }^{(36)}$, including the utilisation of supplementary nutrition ${ }^{(37)}$. Women's parity was observed to influence the consumption of IFA and SF in opposite directions, where higher parity women were less likely to consume IFA and more likely to receive SF in contrast to primiparous women. Similar patterns of IFA consumption have been noted in other studies ${ }^{(22)}$, which reflects the higher prevalence of anaemia among multiparous women ${ }^{(38)}$.

Concurrent to the results of other similar studies ${ }^{(34,39)}$, the consumption of IFA was observed to be higher among economically well-off women, although they were less likely to receive SF. Further, women classified under the caste affiliation of OBC, SC and ST were found to be less likely to consume IFA but more likely to receive SF. The low consumption of IFA among women belonging to disadvantaged social classes could be due to lack of awareness regarding IFA's beneficial effects on mother and baby, myths surrounding IFA use, low-risk perception of anaemia, limited availability of resources ${ }^{(40)}$, lower use of ANC services ${ }^{(41)}$ and lesser autonomy at home ${ }^{(42)}$. This highlights the need to increase the outreach of counselling on IFA supplements to pregnant women from targeted social groups. Muslim women were less likely to consume IFA and receive $S F$, which is supported by similar studies ${ }^{(35)}$ identifying that Muslim women often showed restricted utilisation of $\mathrm{MCH}$ services ${ }^{(43)}$. 


\section{Public Health Nutrition}

Table 4 Multilevel binary regression analysis for supplementary food, India, 2015-16

\begin{tabular}{|c|c|c|c|c|c|c|c|c|c|c|c|c|}
\hline \multirow[b]{2}{*}{ Background variables } & \multicolumn{4}{|c|}{ Model 1 (individual/household level) } & \multicolumn{4}{|c|}{ Model 2 (model 1 + programme level) } & \multicolumn{4}{|c|}{ Model 3 (model $2+$ contextual level) } \\
\hline & OR & SE & $95 \% \mathrm{Cl}$ & $P$ & OR & SE & $95 \% \mathrm{Cl}$ & $P$ & OR & SE & $95 \% \mathrm{Cl}$ & $P$ \\
\hline \multicolumn{13}{|l|}{ Fixed effect } \\
\hline \multicolumn{13}{|l|}{ Individual/household factors } \\
\hline \multicolumn{13}{|l|}{ Age at childbirth } \\
\hline$\leq 19$ (ref.) & 1.00 & & & & 1.00 & & & & 1.00 & & & \\
\hline $20-24$ & 1.09 & & $1.04,1.14$ & 0.001 & 1.07 & & $1.02,1.13$ & 0.011 & 1.07 & & $1.01,1.13$ & 0.012 \\
\hline $25-29$ & 0.98 & & $0.93,1.04$ & 0.484 & 0.97 & & $0.92,1.03$ & 0.295 & 0.98 & & $0.93,1.04$ & 0.500 \\
\hline $30-34$ & 0.90 & & $0.85,0.96$ & 0.001 & 0.90 & & $0.84,0.96$ & 0.001 & 0.92 & & $0.86,0.98$ & 0.009 \\
\hline $35-39$ & 0.84 & & $0.77,0.91$ & $<0.001$ & 0.85 & & $0.78,0.93$ & $<0.001$ & 0.87 & & $0.80,0.95$ & 0.001 \\
\hline $40-49$ & 0.76 & & $0.67,0.85$ & $<0.001$ & 0.84 & & $0.74,0.95$ & 0.004 & 0.85 & & $0.75,0.96$ & 0.009 \\
\hline \multicolumn{13}{|l|}{ Women's education } \\
\hline No schooling (ref.) & 1.00 & & & & 1.00 & & & & 1.00 & & & \\
\hline$<5$ years & $1 \cdot 11$ & & $1.05,1.17$ & $<0.001$ & 1.04 & & $0.98,1.10$ & 0.165 & 1.03 & & $0.98,1.10$ & 0.254 \\
\hline $5-7$ years & 1.18 & & $1 \cdot 14,1.23$ & $<0.001$ & 1.09 & & $1.05,1.14$ & $<0.001$ & 1.07 & & $1.02,1.12$ & 0.002 \\
\hline $8-9$ years & 1.23 & & $1.18,1.29$ & $<0.001$ & $1 \cdot 12$ & & $1.07,1 \cdot 17$ & $<0.001$ & 1.08 & & $1.03,1.13$ & 0.001 \\
\hline $10-11$ years & $1 \cdot 13$ & & $1.08,1 \cdot 19$ & $<0.001$ & 1.02 & & $0.97,1.08$ & 0.410 & 0.99 & & $0.94,1.04$ & 0.654 \\
\hline$\geq 12$ years & 0.94 & & $0.90,0.98$ & 0.009 & 0.88 & & $0.84,0.93$ & $<0.001$ & 0.86 & & $0.82,0.90$ & $<0.001$ \\
\hline \multicolumn{13}{|l|}{ Parity } \\
\hline 1 (ref.) & 1.00 & & & & 1.00 & & & & 1.00 & & & \\
\hline $2-3$ & 1.13 & & $1 \cdot 10,1 \cdot 17$ & $<0.001$ & $1 \cdot 14$ & & $1 \cdot 10,1 \cdot 18$ & $<0.001$ & $1 \cdot 14$ & & $1 \cdot 10,1 \cdot 18$ & $<0.001$ \\
\hline $4-5$ & 1.21 & & $1 \cdot 16,1 \cdot 27$ & $<0.001$ & 1.24 & & $1 \cdot 18,1.30$ & $<0.001$ & 1.24 & & $1.17,1.30$ & $<0.001$ \\
\hline$\geq 6$ & 1.19 & & $1 \cdot 10,1 \cdot 28$ & $<0.001$ & 1.26 & & $1.16,1.36$ & $<0.001$ & 1.25 & & $1.16,1.35$ & $<0.001$ \\
\hline \multicolumn{13}{|c|}{ Gender composition of living children } \\
\hline No sons (ref.) & 1.00 & & & & 1.00 & & & & 1.00 & & & \\
\hline At least one son & 0.98 & & $0.95,1.01$ & 0.133 & 0.99 & & $0.96,1.02$ & 0.404 & 0.99 & & $0.96,1.02$ & 0.413 \\
\hline \multicolumn{13}{|l|}{ Wealth quintile } \\
\hline Poorest (ref.) & 1.00 & & & & 1.00 & & & & 1.00 & & & \\
\hline Poorer & 1.04 & & $1.00,1.08$ & 0.056 & 1.00 & & $0.96,1.04$ & 0.896 & 1.02 & & $0.98,1.06$ & 0.447 \\
\hline Middle & 0.93 & & $0.89,0.98$ & 0.002 & 0.92 & & $0.88,0.96$ & 0.001 & 0.99 & & $0.94,1.04$ & 0.738 \\
\hline Richer & 0.70 & & $0.67,0.74$ & $<0.001$ & 0.73 & & $0.69,0.77$ & $<0.001$ & 0.86 & & $0.81,0.91$ & $<0.001$ \\
\hline Richest & 0.39 & & $0.37,0.41$ & $<0.001$ & 0.45 & & $0.42,0.48$ & $<0.001$ & 0.59 & & $0.55,0.63$ & $<0.001$ \\
\hline \multicolumn{13}{|l|}{ Caste } \\
\hline Others (ref.) & 1.00 & & & & 1.00 & & & & 1.00 & & & \\
\hline Scheduled Castes & 1.66 & & $1.59,1.74$ & $<0.001$ & 1.54 & & $1.47,1.62$ & $<0.001$ & 1.56 & & $1.49,1.64$ & $<0.001$ \\
\hline Scheduled Tribes & 1.46 & & $1.37,1.55$ & $<0.001$ & 1.39 & & $1.30,1.47$ & $<0.001$ & 1.36 & & $1.28,1.45$ & $<0.001$ \\
\hline Other Backward Classes & 1.36 & & $1.31,1.42$ & $<0.001$ & $1 \cdot 31$ & & $1.26,1.36$ & $<0.001$ & 1.31 & & $1.26,1.36$ & $<0.001$ \\
\hline \multicolumn{13}{|l|}{ Religion } \\
\hline Hindu (ref.) & 1.00 & & & & 1.00 & & & & 1.00 & & & \\
\hline Muslim & 0.75 & & $0.72,0.79$ & $<0.001$ & 0.76 & & $0.72,0.80$ & $<0.001$ & 0.83 & & $0.79,0.87$ & $<0.001$ \\
\hline Others & 0.96 & & $0.89,1.02$ & 0.197 & 0.97 & & $0.90,1.04$ & 0.352 & 0.96 & & $0.90,1.03$ & 0.303 \\
\hline \multicolumn{13}{|c|}{ Programme factors } \\
\hline \multicolumn{13}{|c|}{ Prenatal care timing (months of pregnancy) } \\
\hline No ANC (ref.) & 1.00 & & & & 1.00 & & & & 1.00 & & & \\
\hline Early ( $\leq 3$ months) & & & & & 1.42 & & $1.16,1.72$ & 0.001 & 1.41 & & $1.16,1.72$ & 0.001 \\
\hline Intermediate (4-5 months) & & & & & 1.40 & & $1 \cdot 15,1 \cdot 71$ & 0.001 & 1.40 & & $1.14,1.70$ & 0.001 \\
\hline Late ( $\geq 6$ months) & & & & & 1.23 & & $1.01,1.51$ & 0.043 & 1.22 & & $1.00,1.49$ & 0.053 \\
\hline
\end{tabular}




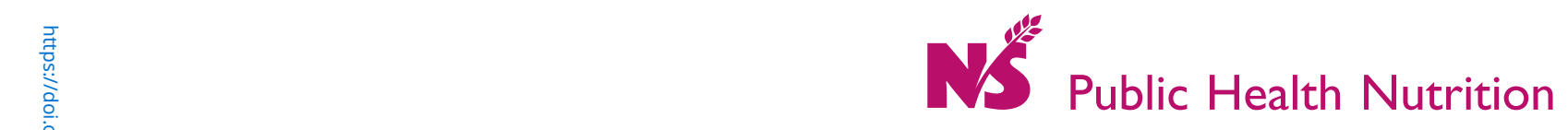

Table 4 Continued

\begin{tabular}{|c|c|c|c|c|c|c|c|c|c|c|c|c|}
\hline \multirow[b]{2}{*}{ Background variables } & \multicolumn{4}{|c|}{ Model 1 (individual/household level) } & \multicolumn{4}{|c|}{ Model 2 (model $1+$ programme level) } & \multicolumn{4}{|c|}{ Model 3 (model $2+$ contextual level) } \\
\hline & OR & SE & $95 \% \mathrm{Cl}$ & $P$ & OR & SE & $95 \% \mathrm{Cl}$ & $P$ & OR & SE & $95 \% \mathrm{Cl}$ & $P$ \\
\hline \multicolumn{13}{|l|}{ Total prenatal visits } \\
\hline$<4$ (ref.) & & & & & 1.00 & & & & 1.00 & & & \\
\hline$\geq 4$ & & & & & 1.07 & & $1.04,1.11$ & $<0.001$ & 1.08 & & $1.05,1.12$ & $<0.001$ \\
\hline \multicolumn{13}{|l|}{ Met healthcare providers during pregnancy } \\
\hline Not met (ref.) & & & & & 1.00 & & & & 1.00 & & & \\
\hline Met at home only & & & & & $5 \cdot 36$ & & $5 \cdot 16,5 \cdot 56$ & $<0.001$ & $5 \cdot 17$ & & $4.98,5.37$ & $<0.001$ \\
\hline Met elsewhere only & & & & & $4 \cdot 14$ & & $4 \cdot 00,4 \cdot 28$ & $<0.001$ & 4.05 & & $3.91,4.19$ & $<0.001$ \\
\hline Met at both places & & & & & $6 \cdot 17$ & & $5.86,6.49$ & $<0.001$ & 5.99 & & $5.69,6.30$ & $<0.001$ \\
\hline \multicolumn{13}{|l|}{ Place of ANC received } \\
\hline At home only (ref.) & & & & & 1.00 & & & & 1.00 & & & \\
\hline Government hospital only & & & & & 1.02 & & $0.96,1.09$ & 0.524 & 1.06 & & $1.00,1.13$ & 0.057 \\
\hline $\mathrm{CHC} /$ rural hospital/block PHC only & & & & & 1.26 & & $1 \cdot 18,1.35$ & $<0.001$ & 1.26 & & $1.18,1.35$ & $<0.001$ \\
\hline PHC only & & & & & $1 \cdot 18$ & & $1.09,1.27$ & $<0.001$ & $1 \cdot 14$ & & $1.06,1.24$ & 0.001 \\
\hline ICDS/SC only & & & & & 1.42 & & $1.29,1.56$ & $<0.001$ & 1.37 & & $1.25,1.51$ & $<0.001$ \\
\hline Private hospital/clinic only & & & & & 0.71 & & $0.67,0.76$ & $<0.001$ & 0.72 & & $0.68,0.77$ & $<0.001$ \\
\hline At least government hospital & & & & & 1.07 & & $0.99,1.17$ & 0.104 & $1 \cdot 10$ & & $1.01,1.20$ & 0.025 \\
\hline At least $\mathrm{CHC} /$ rural hospital/block PHC & & & & & 1.29 & & $1.12,1.49$ & 0.001 & 1.29 & & $1.12,1.49$ & 0.001 \\
\hline At least PHC & & & & & 1.27 & & $1 \cdot 10,1.47$ & 0.001 & 1.22 & & $1.06,1.41$ & 0.007 \\
\hline At least ICDS/SC & & & & & $2 \cdot 02$ & & $1 \cdot 89,2 \cdot 15$ & $<0.001$ & 1.97 & & $1 \cdot 85,2 \cdot 10$ & $<0.001$ \\
\hline Private hospital/clinic & & & & & $1 \cdot 13$ & & $1.05,1.20$ & 0.001 & $1 \cdot 12$ & & $1.05,1.20$ & 0.001 \\
\hline No ANC & & & & & 1.02 & & $0.83,1.25$ & 0.869 & 1.01 & & $0.83,1.24$ & 0.895 \\
\hline \multicolumn{13}{|l|}{ Compositional/contextual factors } \\
\hline \multicolumn{13}{|l|}{ Proportion of illiterate women in PSU (\%) } \\
\hline $0-25$ (ref.) & & & & & & & & & 1.00 & & & \\
\hline $25-50$ & & & & & & & & & 1.01 & & $0.96,1.07$ & 0.717 \\
\hline $50-75$ & & & & & & & & & 0.94 & & $0.89,1.00$ & 0.055 \\
\hline $75-100$ & & & & & & & & & 0.82 & & $0.76,0.88$ & $<0.001$ \\
\hline \multicolumn{13}{|l|}{ Proportion of poor women in PSU (\%) } \\
\hline $0-25$ (ref.) & & & & & & & & & 1.00 & & & \\
\hline $25-50$ & & & & & & & & & 1.15 & & $1.08,1.22$ & $<0.001$ \\
\hline $50-75$ & & & & & & & & & 1.15 & & $1.08,1.24$ & $<0.001$ \\
\hline $75-100$ & & & & & & & & & $1 \cdot 11$ & & $1.03,1.20$ & 0.010 \\
\hline \multicolumn{13}{|l|}{ Pregnancies registered in the district (\%) } \\
\hline $0-25$ (ref.) & & & & & & & & & 1.00 & & & \\
\hline $25-50$ & & & & & & & & & $3 \cdot 20$ & & $1.88,5.44$ & $<0.001$ \\
\hline $50-75$ & & & & & & & & & 12.59 & & $7.54,21.03$ & $<0.001$ \\
\hline $75-100$ & & & & & & & & & 8.01 & & $4.46,14.39$ & $<0.001$ \\
\hline \multicolumn{13}{|l|}{ Place of current residence } \\
\hline Rural (ref.) & & & & & & & & & 1.00 & & & \\
\hline Urban & & & & & & & & & 0.44 & & $0.42,0.47$ & $<0.001$ \\
\hline \multicolumn{13}{|l|}{ Region } \\
\hline Non-High Focus States (ref.) & & & & & & & & & 1.00 & & & \\
\hline High Focus States & & & & & & & & & 1.87 & & $1.55,2 \cdot 24$ & $<0.001$ \\
\hline \multicolumn{13}{|l|}{ Random-effects parameters } \\
\hline District variance & $2 \cdot 012$ & 0.118 & $1 \cdot 79,2 \cdot 26$ & & 1.472 & 0.088 & $1.31,1.65$ & & 1.033 & 0.062 & $0.92,1.16$ & \\
\hline PSU variance & 1.287 & 0.025 & $1.24,1.34$ & & 1.036 & 0.023 & $0.99,1.08$ & & 0.979 & 0.022 & $0.94,1.02$ & \\
\hline
\end{tabular}




\section{Programme factors}

Availing ANC during early pregnancy (within the first trimester) is a key to better utilisation of reproductive health services in continuum. This was observed to have a positive influence on IFA consumption and SF receipt. Similarly, making four or more prenatal visits as recommended had a positive influence on the utilisation of both IFA and SF. Parallel studies have found prenatal visits to be associated with improved awareness and consequent utilisation of IFA ${ }^{(44)}$.

When examining the place of prenatal care, women who received ANC at any government healthcare institution were more likely to consume IFA and receive SF than those who received ANC at home, which is supported by other studies ${ }^{(45)}$. The highest odds of consuming IFA and receiving SF was recorded among women who met with healthcare providers at home or elsewhere compared to those who did not meet at all.

\section{Contextual factors}

The influence of aggregated literacy among women in the community (e.g. village) was considerable in the utilisation of both maternal nutritional interventions. Education has been underlined by multiple studies to define health behaviour $^{(46)}$ and is considered one of the most influential factors in the utilisation of maternal healthcare services in developing countries ${ }^{(47)}$. Therefore, without formal education, there is a higher chance of women in the community not being aware of the services, or developing misinformed perceptions about the same. On the other hand, the aggregated poverty of the community (at the PSU level) did not show a significant influence on the consumption of IFA, but this was influential in terms of the utilisation of SF. Socioeconomic status could influence the utilisation of ICDS services; for instance, it has been observed that the participation of upper- and middle-income households remains lower in availing SF-alike services ${ }^{(48)}$.

Concurrent to the findings relating to pregnancy registration and utilisation of supplements ${ }^{(20,44,49)}$, we found that as the percentage of pregnancy registrations increased in the district, IFA and SF utilisation increased, too. The utilisation of SF was likely to be higher among women belonging to rural households than their urban counterparts. As the distribution of SF was planned through an anganwadi, which is primarily a rural functional centre for women and childcare, proximity to the centre might possibly influence the utilisation of this particular ICDS service.

Women in the HFS were less likely to consume IFA and more likely to receive SF compared to those in the NHFS, while the opposite behaviour was observed in terms of the utilisation of SF. However, a further analysis revealed that women residing in NHFS, compared to those residing in HFS, had higher odds of consuming IFA100 if they had gone for their first prenatal check-up within 3 months of pregnancy and subsequently made four or more prenatal 
visits. The odds of consuming IFA100 increased almost thirty times among women in NHFS and about six times in HFS if the coverage of pregnancy registration exceeded three-fourth (compared to under one-fourth) in the district. Adherence to mandated IFA dosage has been found to be poor among women in $\mathrm{HFS}^{(34)}$, which could be understood in terms of infrastructural and evaluative lacunae faced by healthcare institutions ${ }^{(50)}$ in these states.

This study found a better utilisation of SF in NHFS among women of higher parity, women who made an early prenatal care visit, women who went for four or more prenatal care visits and women who visited ICDS centres or sub-centres at least once in order to receive ANC. A higher proportion of illiterate women in the community in both HFS and NHFS showed a less likelihood of receiving SF. Similar to IFA uptake, a higher proportion of pregnancy registration in the district favoured women receiving SF, especially in HFS. Results also suggested that women from a community in HFS with a higher proportion of poverty were more likely to receive SF. Despite the infrastructural deficits observed by several studies ${ }^{(51)}$,the increasing likelihood of women receiving SF indicated the popularity of free meals provided by anganwadis in HFS. However, the lower likelihood of women receiving SF in urban areas calls for a further exploration and implementation of relevant focussed interventions in both HFS and NHFS.

\section{Study limitations}

This study is based on an analysis of a large-scale multiaspect cross-sectional data, which was not specifically designed to explore the utilisation of nutritional health programmes. Owing to its cross-sectional design, the possibility of exploring causation among variables is limited, while the use of self-reported data poses a possibility of over- or underestimation of outcomes attributable to recall errors. Although the variables were selected based on a thorough literature review and expert consultations, the amount of unobserved heterogeneity still left unexplained provides scope for future studies to incorporate more salient variables not captured by the NFHS. Moreover, based on the information given in the NFHS, it is not known whether the women received IFA tablets or syrup under the public health programmes or purchased by themselves. However, the objective of appraising the utilisation patterns of IFA and SF as maternal nutrition interventions is fulfilled to a large extent by this study.

\section{Conclusion}

This comprehensive cross-sectional study appraising the two pertinent public health interventions to improve maternal nutrition in India is of significant interest in terms of accounting for the observed potential socioeconomic, programmatic and contextual factors. The application of multilevel analysis also provided an opportunity to identify the administrative units where district-level contextual factors such as supply-side system-level functionaries could be responsible for undermining the utilisation of nutrition interventions. The study showed that the consumption of a recommended dosage of IFA improved with a better utilisation of prenatal health services, especially in communities where pregnancies were registered in higher proportions, when women regularly interacted with community health workers or healthcare providers during pregnancy, when women received their first prenatal check-up early in their pregnancy followed by more (at least four) such check-ups. Women's education and their economic status were obvious favourable factors for IFA consumption. Similarly, the likelihood of receiving SF from an anganwadi/ICDS centre was higher among women having a regular contact with healthcare providers and making more number of prenatal visits, at least once at an ICDS centre or sub-centre. Women living in rural areas accounting for a higher registration of pregnancies and a higher proportion of households with poor economic status were more likely to receive SF. Stark differences in the utilisation of both maternal nutrition interventions within and between districts and between HFS and NHFS were evident in this study. Our results also revealed a considerable unobserved heterogeneity at community and district levels, even after controlling for a range of relevant potential factors. Repeated, focussed and concurrent monitoring as well as improvised implementation of public provisioning systems (such as community outreach programmes conducted by peripheral sub-centres, Village Health Nutrition Day, etc.) in the targeted areas would be a key for effective, sustainable and enhanced public health nutrition programmes in diverse community settings such as in India.

\section{Acknowledgements}

Acknowledgements: During the preparation of this article, the first author (P.K.S.) was a Max Planck India Mobility Fellow (M.PG.A.DEFO0002) at the Max Planck Institute for Demographic Research, Rostock, Germany. The authors thank anonymous reviewers for their helpful comments and feedback on an earlier draft of this article. We also thank the Demographic and Health Survey of India for providing the NFHS-4 micro datasets. Financial support: This study received no specific grant from any funding agency, commercial or not-for-profit sectors. Conflict of interest: The authors declare that there is no conflict of interest. Authorship: P.K.S., L.S. and C.K. conceptualised the study. P.K.S. and L.S. analysed the data. P.K.S., R.D., C.K. and R.K.R. wrote the article. All authors critically revised the article and approved the final manuscript. Ethics of human subject participation: The present study is based on anonymised NFHS data available in the public domain. Thus, no separate ethical clearance was required for this study. 


\section{Supplementary material}

For supplementary material accompanying this article visit https://doi.org/10.1017/S1368980020001007

\section{References}

1. Black RE, Victora CG, Walker SP et al. (2013) Maternal and child undernutrition and overweight in low-income and middle-income countries. Lancet 382, 427-451.

2. McLean E, Cogswell M, Egli I et al. (2009) Worldwide prevalence of anaemia, WHO vitamin and mineral nutrition information system, 1993-2005. Public Health Nutr 12, 444-454.

3. Beal T, Massiot E, Arsenault JE et al. (2017) Global trends in dietary micronutrient supplies and estimated prevalence of inadequate intakes. PLoS One 12, e0175554.

4. Development Initiatives (2018) 2018 Global Nutrition Report: Shining a Light to Spur Action on Nutrition. Bristol: Development Initiatives.

5. Ramakrishnan U, Imhoff-Kunsch B \& Martorell R (2014) Maternal nutrition interventions to improve maternal, newborn, and child health outcomes. Nestle Nutr Inst Workshop Ser 78, 71-80.

6. Dandona L, Dandona R, Kumar GA et al. (2017) Nations within a nation: variations in epidemiological transition across the states of India, 1990-2016 in the Global Burden of Disease Study. Lancet 390, 2437-2460.

7. International Institute for Population Sciences (IIPS) and ICF (2017) National Family Health Survey (NFHS-4), 2015-16. Mumbai: International Institute for Population Sciences (IIPS).

8. Kotwal A, Murugkar M \& Ramaswami B (2013) Some reflections on the National Food Security Act. Yojana 57, 25-29.

9. Rai RK, Fawzi WW, Barik A et al. (2018) The burden of irondeficiency anaemia among women in India: how have iron and folic acid interventions fared? WHO South East Asia J Public Health 7, 18.

10. Harika R, Faber M, Samuel F et al. (2017) Micronutrient status and dietary intake of iron, vitamin A, iodine, folate and zinc in women of reproductive age and pregnant women in Ethiopia, Kenya, Nigeria and South Africa: a systematic review of data from 2005 to 2015. Nutrients $\mathbf{9}$ 1096.

11. Wiradnyani LAA, Khusun H, Achadi EL et al. (2016) Role of family support and women's knowledge on pregnancyrelated risks in adherence to maternal iron-folic acid supplementation in Indonesia. Public Health Nutr 19 , 2818-2828.

12. Raut MK, Reddy JC \& Rahman MA (2018) Association between adherence to iron intake and anaemia prevalence among women in Cambodia and India: new evidence from recent global demographic and health surveys. Int $J$ Community Med Public Health 5, 3061-3073.

13. Varghese JS, Swaminathan S, Kurpad AV et al. (2019) Demand and supply factors of iron-folic acid supplementation and its association with anaemia in North Indian pregnant women. PLoS One 14, e0210634.

14. Drèze J \& Khera R (2017) Recent social security initiatives in India. World Dev 98, 555-572.

15. Chakrabarti S, Raghunathan K, Alderman H et al. (2019) India's integrated child development services programme; equity and extent of coverage in 2006 and 2016. Bull World Health Organ 97, 270.

16. World Health Organization (2010) A Conceptual Framework for Action on the Social Determinants of Health. Geneva:
World Health Organization. https://www.who.int/ social_determinants/resources/csdh_framework_action_05_ 07.pdf (accessed January 2020).

17. World Health Organization (2012) Daily Iron and Folic Acid Supplementation in Pregnant Women: Guideline. Geneva: World Health Organization.

18. Government of India (2009) Revised Nutritional and Feeding Norms for Supplementary Nutrition in ICDS Scheme. New Delhi: Ministry of Women and Child Development, Government of India. https://icds-wcd.nic.in/univ_icds/ univ_icds5.pdf (accessed January 2020).

19. Selvaraj K, Arumugasamy P \& Sarkar S (2017) Compliance and patterns of iron-folic acid intake among adolescent girls and antenatal women in rural Tamil Nadu. CHRISMED J Health Res 4, 87 .

20. Warvadekar K, Reddy JC, Sharma S et al. (2018) Sociodemographic and economic determinants of adherence to iron intake among pregnant women in selected low and lower middle income countries in Asia: insights from a cross-country analyses of global demographic and health surveys. Int J Community Med Public Health 5, 1552-1569.

21. Kavle JA \& Landry M (2018) Community-based distribution of iron-folic acid supplementation in low-and middle-income countries: a review of evidence and programme implications. Public Health Nutr 21, 346-354.

22. Alam A, Rasheed S, Khan NUZ et al. (2015) How can formative research inform the design of an iron-folic acid supplementation intervention starting in first trimester of pregnancy in Bangladesh? BMC Public Health 15, 374.

23. Subramanian SV, Chen JT, Rehkopf DH et al. (2006) Comparing individual-and area-based socioeconomic measures for the surveillance of health disparities: a multilevel analysis of Massachusetts births, 1989-1991. Am J Epidemiol 164, 823-834.

24. Stephenson R, Baschieri A, Clements S et al. (2006) Contextual influences on the use of health facilities for childbirth in Africa. Am J Public Health 96, 84-93.

25. Coleman JS (1990) Foundations of Social Theory. Cambridge, MA: Harvard University Process.

26. Das TK \& Teng BS (1998) Between trust and control: developing confidence in partner cooperation in alliance. Acad Manag Rev 23, 491-512.

27. Gage AJ \& Guirlène Calixte M (2006) Effects of the physical accessibility of maternal health services on their use in rural Haiti. Popul Stud 60, 271-288.

28. Babalola S \& Fatusi A (2009) Determinants of use of maternal health services in Nigeria-looking beyond individual and household factors. BMC Pregnancy Childbirth 9, 43.

29. Agresti A \& Kateri M (2011) Categorical Data Analysis. Berlin, Heidelberg: Springer.

30. Goldstein H (2011) Multilevel Statistical Models, vol. 922. Hoboken, NJ: John Wiley \& Sons.

31. Goldstein H \& Healy MJR (1995) The graphical presentation of a collection of means. I R Stat Soc Ser A 158, 175-177.

32. StataCorp LP (2015) Stata Statistical Software: Release 14. College Station, TX: StataCorp LP.

33. Subramanian SV, Duncan C \& Jones K (2001) Multilevel perspectives on modeling census data. Environ Plan A 33, 399-417.

34. Chourasia A, Pandey CM \& Awasthi A (2017) Factors influencing the consumption of iron and folic acid supplementations in high focus states of India. Clin Epidemiol Glob Health 5, 180-184.

35. Wendt A, Stephenson R, Young M et al. (2015) Individual and facility-level determinants of iron and folic acid receipt and adequate consumption among pregnant women in rural Bihar, India. PLoS One 10, e0120404.

36. Sharma K, Sharma J, Choudhary J et al. (2018) A study to assess the knowledge of mothers regarding reproductive child health programme activity and its utilization in selected 
urban community in Gwalior city. Int J Community Med Public Health 5, 4787-4794.

37. Vishnu CS, Nirgude AS, Rajarathnam A et al. (2019) Do the pregnant mothers utilize supplementary nutrition along with other antenatal services? A cross sectional study from Mangaluru, Karnataka state, India. Int J Community Med Public Health 6, 1614-1617.

38. Viveki RG, Halappanavar AB, Viveki PR et al. (2012) Prevalence of anaemia and its epidemiological determinants in pregnant women. Al Ameen J Med Sci 5, 216-223.

39. Begum S (2012) Factors associated with adherence to iron folic acid supplementations during pregnancy in Uttar Pradesh. Indian J Matern Child Health 14, 8.

40. Nisar YB, Dibley MJ \& Mir AM (2014) Factors associated with non-use of antenatal iron and folic acid supplements among Pakistani women: a cross sectional household survey. BMC Pregnancy Childbirth 14, 305.

41. Balarajan Y, Selvaraj S \& Subramanian S V (2011) Health care and equity in India. Lancet 377, 505-515.

42. Diamond-Smith NG, Gupta M, Kaur M et al. (2016) Determinants of persistent anemia in poor, urban pregnant women of Chandigarh city, North India: a mixed method approach. Food Nutr Bull 37, 132-143.

43. Singh PK, Rai RK, Alagarajan M et al. (2012) Determinants of maternity care services utilization among married adolescents in rural India. PLoS One 7, e31666.
44. Roy MP, Mohan U, Singh SK et al. (2013) Socio-economic determinants of adherence to iron and folic acid tablets among rural ante-natal mothers in Lucknow, India. Natl J Community Med 4, 386-391.

45. Kumar V \& Singh P (2017) How far is universal coverage of antenatal care (ANC) in India? An evaluation of coverage and expenditure from a national survey. Clin Epidemiol Glob Health 5, 1-7.

46. Conner M \& Norman P (2005) Predicting Health Behaviour. Amsterdam, Netherlands: McGraw-Hill Education.

47. Sridharan S, Dey A, Seth A et al. (2017) Towards an understanding of the multilevel factors associated with maternal health care utilization in Uttar Pradesh, India. Glob Health Action 10, 1287493.

48. Rao N \& Kaul V (2018) India's integrated child development services scheme: challenges for scaling up. Child Care Health Dev 44, 31-40.

49. Khan REA \& Raza MA (2013) Maternal Health Care: The Case of Iron Supplementation in India. Lahore, Pakistan: Johar Education Society.

50. Wendt AS, Stephenson R, Young MF et al. (2018) Identifying bottlenecks in the iron and folic acid supply chain in Bihar, India: a mixed-methods study. BMC Health Serv Res 18, 281.

51. Gupta A, Gupta S \& Nongkynrih B (2013) Integrated child development services (ICDS) scheme: a journey of 37 years. Indian J community Health 25, 77-81. 